\title{
A system to measure 3-directional relative displacements for a biomedical application
}

\author{
V. L. Spiering, V. Gass, S. Jeanneret, N. F. de Rooij, K. H. Widmer, B. Zurfluh, E. Morscher
}

Abstract A micro system for measuring the relative displacements between a bone and an implant in a prosthetic hip of a human being has been realized. Novel are the small dimensions of the system combined with the possibility to measure relative displacements in three directions. The system consists of a microsensor body, a mechanical transducer element made by precision mechanics, electronics, and the final package. The realization and test results of a prototype system are presented. Typical ranges for these prototypes are $\pm 500 \mu \mathrm{m}$ for lateral directions and $\pm 50 \mu \mathrm{m}$ in axial direction.

\section{1}

\section{Introduction}

The aim of endoprosthetic implant fixation is osteointegration. In order to enhance its fixation, the surface of the (noncemented) implant has a porous structure. A requirement for bony ingrowth into this porous surface is primary stability of the implant in the host bone. Relative motion between implant and bone exceeding 100 to $120 \mu \mathrm{m}$ will prevent bony ingrowth. For measuring these movements a Bone Implant Displacement System (BIRDS) has been developed.

In this particular case, movements in the joint of a hip were of interest. Figure 1 shows a cross-section of a human hip with an artificial joint. The shifts between the upper part of the joint (A) and the hip itself (B) are to be measured. The BIRD-System is located in the bone and has to be installed via a hole in the hip.

By Petersen et al. (1985), one-dimensional shifts were transformed to membrane deformations, which were recorded

Received: 12 September 1994 / Accepted: 22 September 1994

V. L. Spiering', V. Gass, S. Jeanneret, N. F. de Rooij, K. H. Widmer², B. Zurfluh', E. Morscher ${ }^{2}$

Institute of Microtechnology, University of Neuchâtel

Rue A.L. Brequet 2, $\mathrm{CH}-2000$ Neuchâtel, Switzerland

${ }^{1}$ On leave from: MESA Research Institute, University of Twente, The Netherlands

${ }^{2}$ Orthopädische Universitätsklinik Basel,

Labor für Orthopädische Biomechanik,

CH-4031 Basel, Switzerland

Correspondence to: V. L. Spiering

Ben Kloeck is gratefully acknowledged for his contribution concerning this project. The delivery of samples by ASCOM Microelectronics is highly appreciated by strain gauges. Based on a similar principle a complete three-dimensional measurement system is presented in this paper. The system consists of a microsensor body which senses the displacements between this body and the outer end of a mechanical spring, the mechanical transducer element. Measurements have been carried out with both a silicon micromachined accelerometer and a pressure sensor as sensor body. The ranges for the lateral- and axial direction can be chosen for each system by means of the mechanical spring stiffnesses for the different directions. Typical ranges are $\pm 1 \mathrm{~mm}$ for lateral shifts and $\pm 100 \mu \mathrm{m}$ for axial shifts.

The specifications for this biomedical application are:

- lateral displacement range: $\pm 500 \mu \mathrm{m}$

- max. axial movement: $100 \pm 50 \mu \mathrm{m}$

- resolution: $10 \mu \mathrm{m}$

- measurement speed: ca. $10 \mathrm{~Hz}$

The presented system is designed for testing in a simulation machine. This apparatus contains an artificial hip and the implant itself. Several loads can be applied, to simulate actions like walking or lifting. In this way the geometry of the artificial joint can be optimized by measuring the shifts for variable joints, without a real in-vivo experiment.

\section{2}

\section{Realization}

The microsystem is constructed of a micromechanical silicon pressure sensor (or accelerometer), a spring (commercially fabricated on request), housing and electronics. In principle the accelerometer consists of a mass suspended by four beams, in which piezo-resistive strain gauges are realized by boron-doped silicon, as described by T'schan (1992). The pressure sensor is identical, except for the fact that the mass is not released completely, but etched until a membrane thickness of about $20 \mu \mathrm{m}$, see Kloeck (1989). In that case the result is a bossed membrane with the strain gauges located on the thin membrane, see also Fig. 2. The fabrication sequences of both the accelerometer and the pressure sensor have been described before, and use a bipolar compatible industrial process from Ascom (1993). The pressure sensor was bonded to a glass substrate, which functions as an overrange protection in the negative $z$-direction as well.

On top of the sensor mass, a spring is mounted. The applied springstiffness is in $z$-direction $k_{z} \approx 100 \mathrm{~N} / \mathrm{m}$ and in $x y$-direction $k_{x y} \approx 5 \mathrm{~N} / \mathrm{m}$. The spring has been realized by precision mechanical techniques with the special constraints: $(1)$ a conical 


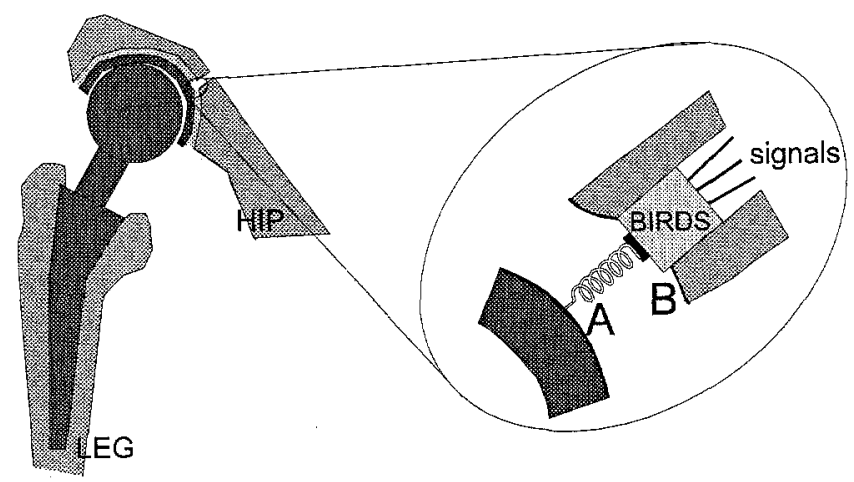

\begin{tabular}{|l|}
\hline Bone \\
Implant \\
Transduction element: spring \\
Deformed structure: \\
beams or thin membrane \\
\hline
\end{tabular}

Fig. 1. Schematic cross-section of an implant in a human hip containing a system to measure relative displacements between point A (implant) and $\mathrm{B}$ (bone)
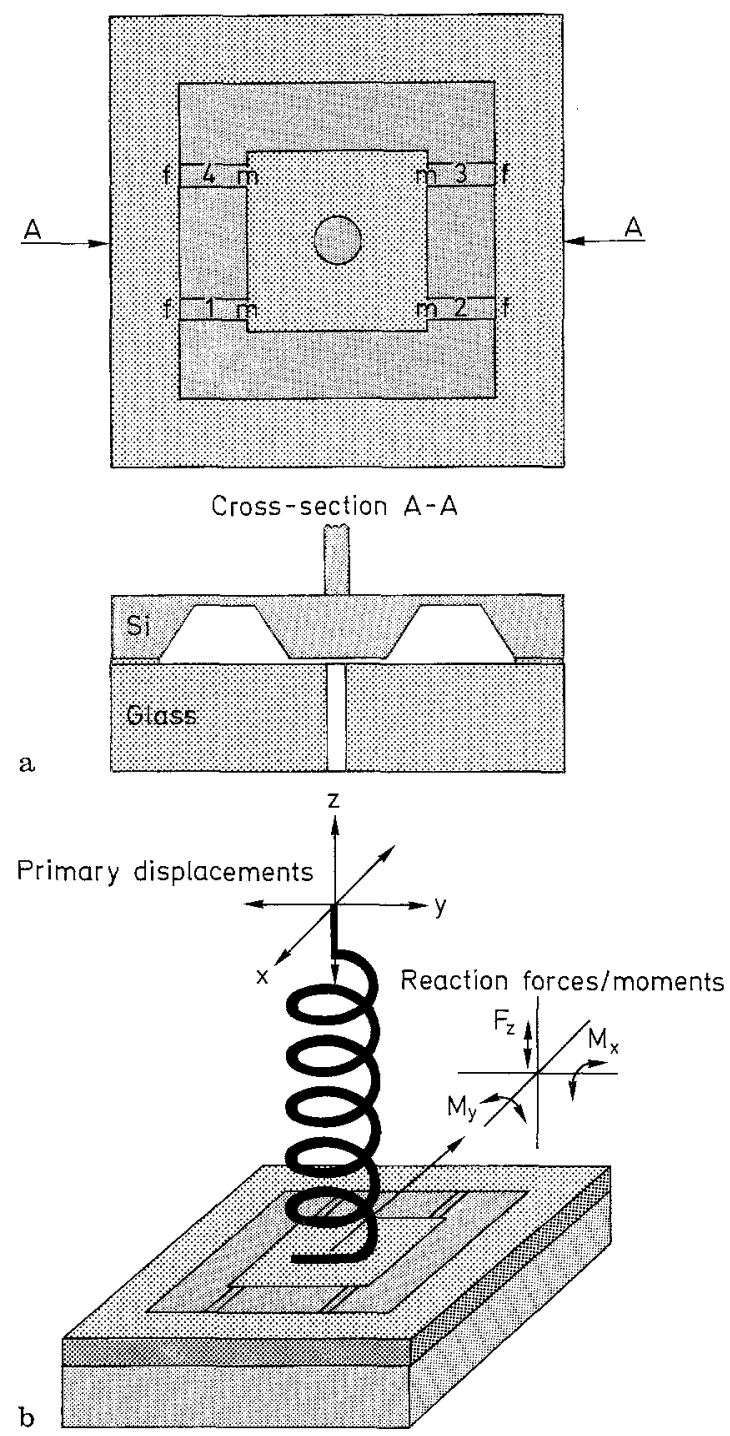

Fig. 2a, b. Schematic drawing, top view and cross-section of the BIRD-System based on the pressure sensor

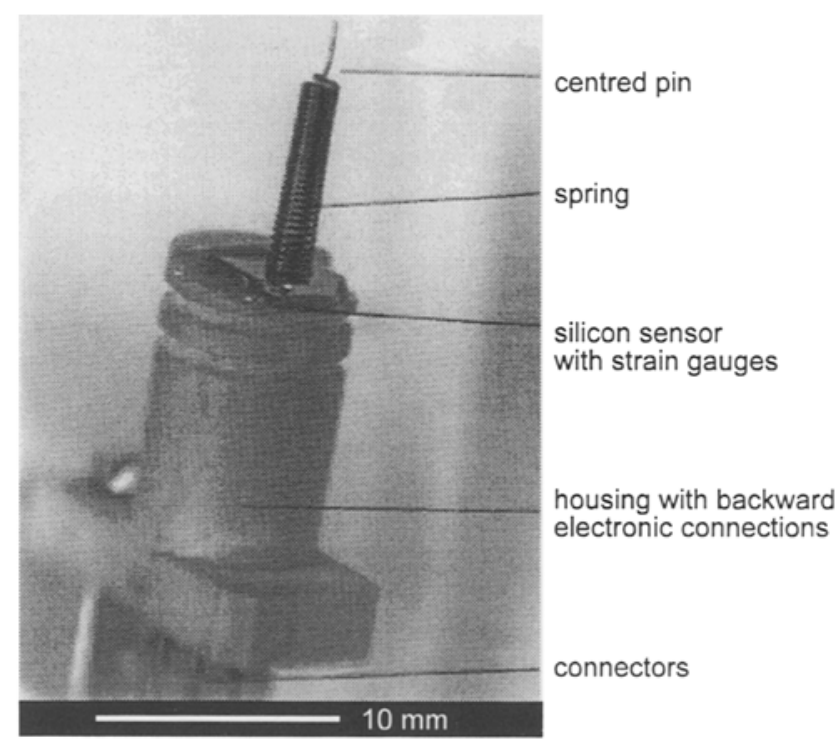

Fig. 3. Photograph of the BIRD-SYSTEM and the housing

overall shape to improve the stability, (2) a flattened ring at the bottom to facilitate the connection on the membrane and (3) a centred pin at the top to allow a proper connection to the implant. By varying the stiffness and length of the spring the measurement range and measure distance can be adjusted easily. Figure 3 shows a photograph of the BIRD-System without package. The system is put on a cylindrical housing with backward electrical connections for installation in the bone. For the final package an aluminum cylinder is fixed on the housing with the spring's end just reaching out.

\section{3}

\section{Operation principle}

By moving the spring's end in $x y$ direction, moments $\left(M_{x}, M_{y}\right)$ are generated on the boss while a $z$-displacement causes a force $\left(F_{z}\right)$ at the centre, see Fig. 2. The resulting deformations are measured by the piezo-resistive strain gauges positioned on the membrane. According to Fig. 4 the locations are numbered from 1 to 4 , corresponding to resistance's $R_{L}$ to $R_{4}$. The positions of the strain gauges are important, because the signals should change independently for the $x, y$ and $z$ displacements. Depending on the position of the resistors on the beam and depending on the beam position there will be an increase $(+)$ or decrease $(-)$ of resistance $\Delta R$. In the realized sensors, resistors $R_{1}$ and $R_{3}$ are placed on the frame (f) and resistors $R_{2}$ and $R_{4}$ on the mass (m). Figure 4 shows the resistance changes for several positive spring movements in case of the realized sensor. In Table 1 the sign of the change of resistance is given for the positive applied spring-movements.

In practice the derivatives of the resistance's to the motions $\partial R_{i} / \partial r$, with $r=(x, y$, or $z)$, will be measured by voltage changes $\partial V_{i} / \partial R_{i} \cdot \partial R_{i} / \partial r$ in (four) Wheatstone-bridge configurations. Because there are four resistance changes, a fourth independent parameter $(\Delta c)$ could be detected. In principle the fourth signal can be used to measure the temperature, because all resistance's 

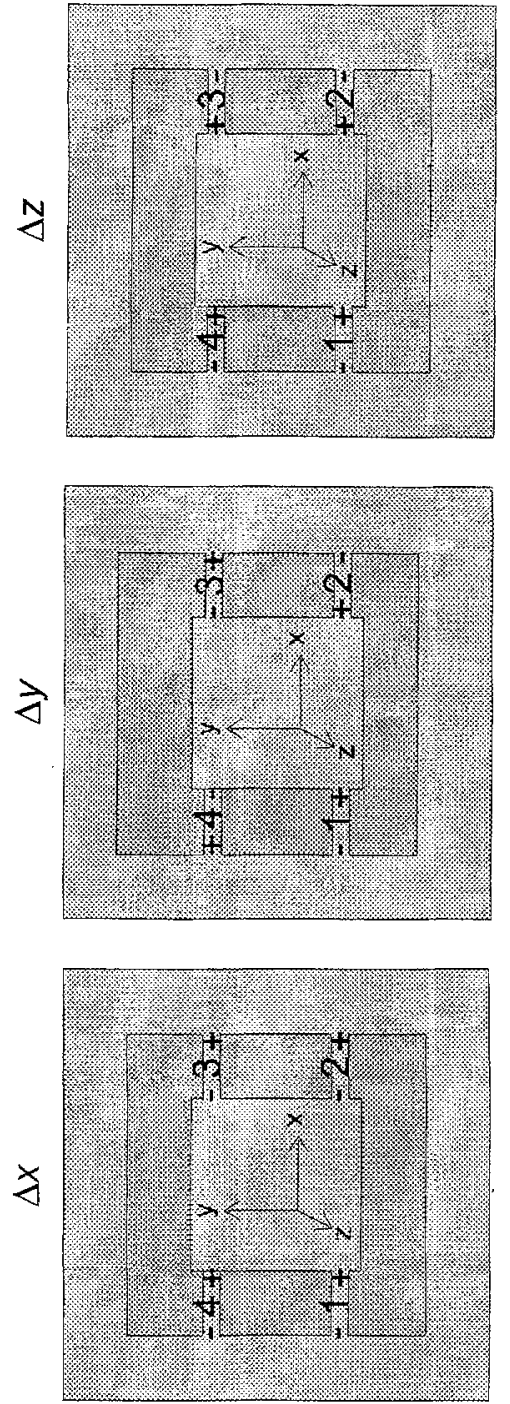

Fig. 4. Sign changes of the resistors for positive spring movements (spring on top)

will change with the same sign. Only one of the resistors of each bridge is actually on-chip so the temperature dependence of the strain gauges is not a filtered common mode signal, as is usually the case for pressure sensors. For the applied prototypes the measurement are done at a constant temperature $\left(20^{\circ} \mathrm{C}\right)$ and with a low voltage supply of the bridge $(1 \mathrm{~V})$. The system is calibrated first by the determination of the matrix elements $A_{i j}$ of the following matrix A:

$\left[\begin{array}{c}\Delta V_{1} \\ \Delta V_{2} \\ \Delta V_{3} \\ \Delta V_{4}\end{array}\right]=\left[\begin{array}{cccc}\frac{\partial V_{1}}{\partial x} & \frac{\partial V_{1}}{\partial y} & \frac{\partial V_{1}}{\partial z} & \frac{\partial V_{1}}{\partial c} \\ \frac{\partial V_{2}}{\partial x} & \frac{\partial V_{2}}{\partial y} & \frac{\partial V_{2}}{\partial z} & \frac{\partial V_{2}}{\partial c} \\ \frac{\partial V_{3}}{\partial x} & \frac{\partial V_{3}}{\partial y} & \frac{\partial V_{3}}{\partial z} & \frac{\partial V_{3}}{\partial c} \\ \frac{\partial V_{4}}{\partial x} & \frac{\partial V_{4}}{\partial y} & \frac{\partial V_{4}}{\partial z} & \frac{\partial V_{4}}{\partial c}\end{array}\right]\left[\begin{array}{c}\Delta x \\ \Delta y \\ \Delta z \\ \Delta c\end{array}\right]$

or $\Delta \bar{V}=A \cdot \Delta \bar{r}$
After this calibration, the position displacements $\Delta \bar{r}$ are detected by measuring the voltage changes $\Delta \bar{V}$ and inversion of $A$ :

$\Delta \bar{r}=A^{-1} \cdot \Delta \bar{V}$

In the measurement range, linearity of the displacements to resistance changes is required, because the matrix coefficients are assumed to be constant. This will be the case if the stiffness of the spring and the silicon membrane are constant in the applied range. The linear range of deformations of the strain gauges together with the given (spring's end) displacement range determine the choice of the springstiffness. The spring cannot be too stiff as the occurring large membrane deformations can cause destruction, and on the other hand a decrease in the spring's stiffness is accompanied by a decrease of sensitivity. For the chosen spring it is important that non-linear deformations are avoided. For a large ratio between spring length and displacement range this linearity is expected, as the deformations are relative small then. The requirement for linearity is not only limited to shifts in one direction, but is also mandatory in case of a superposition of the displacements in different directions (three directional shifts).

\section{4}

\section{Experimental results}

In a calibration set-up, the voltage signal changes in the Wheatstone bridges of the four resistance's were measured as a function of applied displacements in the $x$-, $y$-, and $z$-direction. The spring's end shifts were measured by micrometertranslators. The springstiffnesses are $k_{x}=k_{y}=4.5 \mathrm{~N} / \mathrm{m}$ and $k_{z}=75 \mathrm{~N} / \mathrm{m}$. The spring mass of $30 \mathrm{mg}$ causes an offset corresponding to a spring's end $z$-displacement of circa $4 \mu \mathrm{m}$.

Measurements with the pressure sensor and accelerometer as sensor body were compared. The results were similar, except for an increase of sensitivity by a factor 5 for the accelerometer. This is explained by the lower stiffness of the sensor body: the four beams are more flexible than the closed membrane. However, for reasons of mechanical stability the pressure sensor body was chosen for application in the final BIRD-system.

Results in the range $(500,500,250 \mu \mathrm{m})$ of a BIRD-system containing a pressure sensor as sensor body are presented in Fig. 5. The output voltages are obtained after an amplification of 1000 , and using a constant bridge voltage of $1 \mathrm{~V}$. For all directions within the range there is a good linearity. The main cause for the deviations in these calibrations is the precision of the applied displacements by the micrometer translations (about $1 \mu \mathrm{m}$ ). The measured voltage changes for the different. Wheatstone bridges correspond to the predicted sign changes of the resistors given in Table 1 .

The sensitivities were determined with the best linear fit and are given in matrix notation, as in Eq. (1):

$\left[\begin{array}{l}\Delta V_{1} \\ \Delta V_{2} \\ \Delta V_{3} \\ \Delta V_{4}\end{array}\right]=\frac{1}{1000} \cdot\left[\begin{array}{rrrr}1.895 & 1.494 & 9.932 & 1000 \\ 1.718 & -1.004 & -9.292 & 1000 \\ -1.580 & -1.174 & 9.572 & 1000 \\ -2.088 & 1.256 & -9.932 & 1000\end{array}\right]\left[\begin{array}{c}\Delta x \\ \Delta y \\ \Delta z \\ \Delta c\end{array}\right]$

$\Delta V$ in $V ; \Delta x, \Delta y, \Delta z$ in $\mu \mathrm{m}$; matrix elements in $V / \mu \mathrm{m}$. (1) The coefficients $\Delta V_{i} / \Delta c$ were chosen to be 1. 

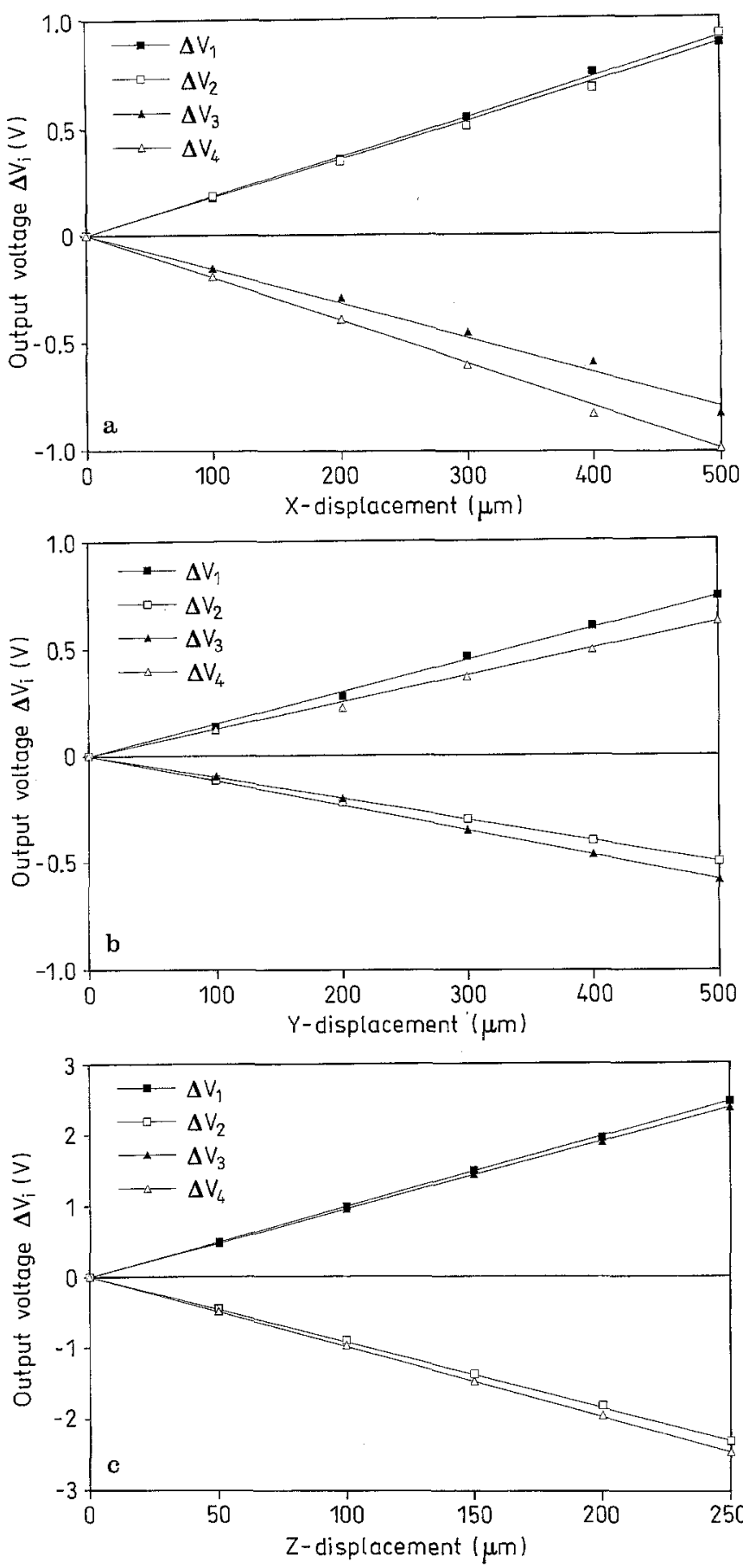

Fig. $5 \mathrm{a}-\mathrm{c}$. Output voltages for the four resistors for applied $x, y$ and $z$-displacements

Table 1. Independent sign changes of the resistors for positive spring movements

\begin{tabular}{lllll}
\hline & \multicolumn{1}{l}{$\Delta R$ by $\Delta x$} & $\Delta R$ by $\Delta y$ & $\Delta R$ by $\Delta z$ \\
& - & - & - \\
$R_{1 \mathrm{ff}}$ & - & - & + & + \\
$R_{2 \mathrm{~m}}$ & - & + & - \\
$R_{3 \mathrm{f}}$ & + & + & + \\
$R_{4 \mathrm{~m}}$ & + & - & + \\
\hline
\end{tabular}
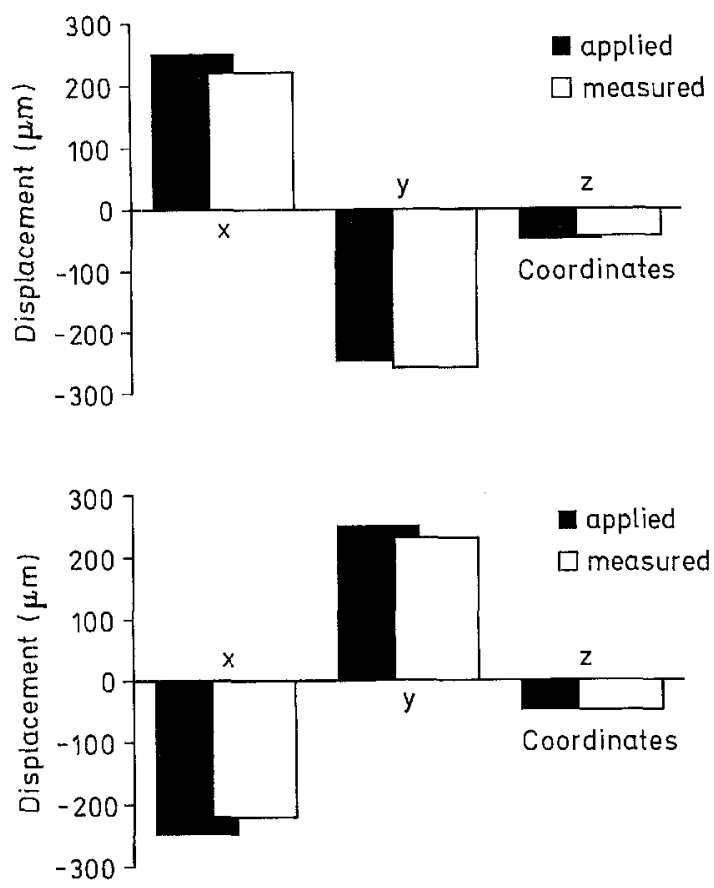

Fig. 6. Comparison of measured and applied $3 \mathrm{D}$-displacements for two different shifts

The average sensitivities of the four gauges for each direction are: $1.82 \pm 15 \%, 1.23 \pm 20 \%$ and $9.68 \pm 4 \% V / \mu \mathrm{m}$. The deviations are mainly caused by the fact that the spring is not exactly centred on the membrane. Since the sensitivities in $z$-direction are less dependent on this asymmetry, differences in this direction are relatively smaller.

The larger sensitivity of $z$-shifts combined with the smaller range (factor 10) cause comparable voltage differences on the full scale for all directions. This prevents the domination of a shift in one direction on the whole signal. The difference in sensitivity between $x$ - and $y$-shifts is due to the asymmetrical location of the strain gauges on the membrane structure, see also Figs. 3 and 4. The structure appears to be a little more sensitive for $x$-translations of the spring.

The inverse of the matrix in eq. (3) is:

$$
\left[\begin{array}{l}
\Delta x \\
\Delta y \\
\Delta z \\
\Delta c
\end{array}\right]=\left[\begin{array}{rrrr}
122 & 152 & -130 & -145 \\
213 & -194 & -210 & 192 \\
23.3 & -28.2 & 28.5 & -23.6 \\
0.220 & 0.282 & 0.276 & 0.222
\end{array}\right]\left[\begin{array}{l}
\Delta V_{1} \\
\Delta V_{2} \\
\Delta V_{3} \\
\Delta V_{4}
\end{array}\right]
$$

With this matrix it is possible to transfer the measured voltage signals into displacements. Figure 6 shows a comparison between applied and measured displacements for a full-space three-directional shift. The applied measurements were detected by the microtranslators. The measured voltage signals were multiplied by the earlier determined calibration matrix to acquire the measured displacement values. The differences are less than $10 \%$ of the measured value. They are caused by the inaccuracy of the set-up and possibly by a small non-linearity in the superposition of the three one-dimensional shifts. 
The realized and tested prototypes BIRD-Systems met the requirements as given in the introduction and they will be applied for testing in a simulation machine in the near future.

\section{5}

\section{Conclusions}

A prototype micro system for measuring relative displacements in three directions between a bone and an implant in a prosthetic hip of a human being has been realized and tested in laboratory conditions successfully. The system contains a micro-mechanical fabricated pressure sensor and a spring made by precision mechanics. After calibration the accuracy of the displacement measurement was better than $10 \%$ of the measured value. Typical ranges for the realized prototypes are $\pm 500 \mu \mathrm{m}$ for lateral directions and $\pm 50 \mu \mathrm{m}$ in axial direction.

\section{References}

ASCOM Microelectronics, Bevaix, Switzerland, 1993

Kloeck, B. (1989) Design, fabrication and characterization of piezoresistive pressure sensors, including the study of electrochemical etch-stop. Ph.D. thesis, Institute of Microtechnology, University of Neuchâtel, Switzerland

Petersen, K.; Kowalski, C.; Brown, J.; Allen, H.; Knutti, J. (1985) A force sensing chip designed for robotic and manufacturing automation applications. Proc. Int. Conf. Solid-State Sensors and Actuators (Transducers ' 85 ), June 11-14 1985, pp 30-32

Tschan, T. (1992) Simulation, design and characterization of a silicon piezoresistive accelerometer, fabricated by a bipolar-compatible industrial process. Ph.D. thesis, Institute of Microtechnology, University of Neuchâtel, Switzerland 